\title{
Journalistische Berufsethik in der Journalistenausbildung
}

\author{
von Jürgen Wilke
}

Wenn man die journalistische Berufsethik zu den Defiziten der Journalistenausbildung zählt ${ }^{1}$, so wird man damit heute großenteils Zustimmung finden. Die Gründe, warum dieses Thema vernachlässigt worden ist, sind vielfältig. Sie liegen zum einen in historisch-gesellschaftlichen Voraussetzungen, sind zum anderen aber - was die Universitäten angeht - vor allem wissenschaftsgeschichtlicher und wissenschaftstheoretischer Art. So lange normative Ansätze in der deutschen Zeitungs- und Publizistikwissenschaft Geltung besaßen, sparte man die ethische Dimension des Journalismus nicht aus. Nirgendwo ist dies deutlicher gewesen als bei Emil Dovifat, für den das Prinzip der Gesinnung in der Publizistik von zentraler Bedeutung war, der vom Journalisten zugleich aber auch Verantwortungsbewußtsein verlangte.

Als solche normativen Ansätze durch die sozialwissenschaftliche Empirie verdrängt wurden, kam nicht nur das Interesse an ethischen Fragen abhanden, sondern es schwand auch die Überzeugung, sich dieses Themas überhaupt wissenschaftlich annehmen zu können. Denn bei kaum einem anderen Thema scheint es schwieriger, zu intersubjektiver Übereinstimmung zu gelangen, als wo es um Ethik geht. Dabei ist diese Unsicherheit der Wissenschaft ein Reflex auf den gewachsenen Wertepluralismus der Gesellschaft. Und damit ist das Problem der Verbindlichkeit aufgeworfen, mit der bestimmte Verhaltensweisen im Journalismus noch begründet und eingefordert werden können.

Schließlich begegnet man gar einem Skeptizismus, ob das moralische Bewußtsein von Journalisten überhaupt »auszubilden « bzw. die Befolgung bestimmter Normen durch

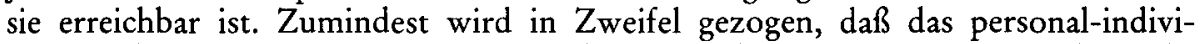
dualistische Postulat, wie es der Ethik nach alter Tradition eigen ist, weiterhilft. Wichtiger als dieses, so wird heute mitunter argumentiert, seien bestimmte strukturelle Vorkehrungen im Mediensystem. ${ }^{3}$

Indessen haben sich in den letzten Jahren auch hierzulande die Anzeichen gemehrt, $\mathrm{da} ß$ man die Vernachlässigung der journalistischen Berufsethik nicht auf sich beruhen lassen sollte. $\mathrm{Zu}$ allerletzt ließe sich dieses Defizit ja aus dem Eindruck rechtfertigen, der Journalismus sei »wertfrei« geworden. Im Gegenteil: Der wissenschaftlichen Abstinenz scheint auf der Seite der Journalisten durchaus ein aus schwer faßbaren Quellen gespeistes normatives Bewußtsein gegenüberzustehen. Im übrigen steht die Vernachlässigung des Themas bei uns in einem auffälligen Kontrast zu der Bedeutung, die die journalistische Berufsethik oder Medienethik in der amerikanischen Journalistenausbildung besitzt. Belegt wird dies durch dortige Erhebungen ${ }^{4}$, aber auch durch eine rege Publikationstätigkeit auf diesem Feld. ${ }^{5}$ Nun mag beides noch nichts über die Effizienz der berufsethischen Unterweisung amerikanischer Journalistik-Studenten besagen. Aber man erkennt darin doch zumindest einen gewissen Reflexionsvorsprung.

Prof. Dr. Jürgen Wilke ist Inhaber des Lehrstuhls für Journalistik I an der Katholischen Universität Eichstätt. 
Daß die Zurückhaltung gegenüber dem Thema journalistische Berufsethik auch bei uns aufgegeben oder zumindest als leichtfertig empfunden wird, dürfte vornehmlich darauf zurückzuführen sein, daß man zunehmend wieder von einer großen Wirkung, ja einer Macht der Massenmedien ausgeht. Denn der Ruf nach der Ethik wird immer dann laut, wenn es um die Bewältigung von Folgeproblemen geht. Insofern steht die Aktualisierung der journalistischen Berufsethik heute in unverkennbarer Parallele zu den Bemühungen um eine (neue) Wissenschaftsethik. ${ }^{6} Z$ war scheinen die Konsequenzen eines ethisch nicht verantworteten wissenschaftlichen Fortschritts weit über das hinauszugehen, was der Journalismus »anrichten « kann. Aber dieser ist längst auch zu einer das gesellschaftliche System bewegenden Kraft geworden, die in ihrer Eigendynamik durchaus dysfunktionale Folgen hervorbringt.

Behauptet man, wie dies hier eingangs geschah, die journalistische Berufsethik gehöre zu den Defiziten der Journalistenausbildung, so ist damit also nichts gerade Neues gesagt. Denn auch in der Bundesrepublik hat es in den letzten Jahren wieder verschiedene Ansätze zur Entwicklung oder Fundierung einer journalistischen Berufsethik gegeben. Der Moraltheologe Alfons Auer machte sich zwar durchaus zum Fürsprecher einer »immanenten Ethik « der Medien: "Im Prozeß der sozialen Kommunikation«, so formulierte er, "handelt sittlich richtig, wer die in diesem Bereich geltenden $\mathrm{Ge}$ setzlichkeiten und die hier gegebenen Sinnziele respektiert. " ' Aber bei aller Offenheit, solche inneren "Gesetzlichkeiten " oder "Sinnziele« zu erkennen, gelangt er kaum über gut gemeinte, aber wenig konkrete und daher unverbindlich anmutende Postulate hinaus ("ehrlicher Makler «, "vollständige Information «, "kritische Solidarität«, "Partnerschaft «). Im Kern bleibt es beim individualethischen Appell, dem eine personale Anthropologie zugrunde liegt.

Um eine dem "Szientismus « entgegengesetzte, "geisteswissenschaftliche» Begründung der journalistischen Berufsethik hat sich Hermann Boventer bemüht. ${ }^{8}$ Er steigt, wie er sagt, in "das Bergwerk der Philosophie "ein, um herauszubrechen, "was an wertvollem Gestein in das Theoriegebäude des Journalismus paßt. ${ }^{9}{ }^{9} \mathrm{Imm}$ geht es darum, die normative Prägung des Journalismus durchsichtig zu machen und »eine reflektierende und nach der Vernünftigkeit ihrer Geltungsgründe fragende Moral « zu entwickeln. Dabei läßt sich seiner Ansicht nach vor allem das Wahrheitspostulat nicht umgehen. Zwar zitiert Boventer nicht nur eine Vielzahl moralischer Autoritäten, sondern hat auch manche Erkenntnisse der jüngeren Kommunikationswissenschaft rezipiert und verarbeitet. Dennoch bleibt im Endergebnis trotz vieler Worte schwer faßbar, welchen praktisch-substantiellen Gehalt die theoretische Grundlegung besitzt.

Sich von solchen Ansätzen und von der gesinnungsethischen Tradition des eigenen Faches absetzend, haben Manfred Rühl und auch Ulrich Saxer versucht, die journalistische Berufsethik kommunikationswissenschaftlich zu begründen. ${ }^{11}$ Journalismus besteht nach Rühl nicht aus Personen, sondern ist ein komplexes soziales Handlungssystem. Insofern sind ihm gegenüber das appellative Pathos einer Individualethik und die Berufung auf einen Satz vorab formulierter Sollensvorschriften unangemessen. Eine Ethik, die der Systemrationalität gerecht werden will, muß vielmehr die institutionalisierten Entscheidungsstrukturen des Journalismus in Rechnung stellen, d.h. die Erwartungen, Rollen, Funktionen und Regeln, die den Prozeß der Herstellung von Öffentlichkeit für Themen steuern. Unter Hinweis darauf, daß zwischen Journalismus und Publikum kein eingleisiges Verhältnis besteht, wird aus der Theorie des symbolischen Interaktionismus schließlich die »Achtung " als zentrale berufsethische 
Kategorie abgeleitet. ${ }^{12}$ Schon Boventer hat - wie ich meine, nicht ohne Grund - hier von einer merkwürdigen Kehrtwendung ${ }^{13}$ in der Argumentation gesprochen. Abgesehen davon, daß das Prinzip der "Achtung « etwas willkürlich isoliert erscheint, ist bemerkenswert, daß auch Rühl letztlich nicht ganz von der Person absehen kann. Einerseits behauptet er zwar, »der Journalist, der als Person nicht Bestandteil des Journalismus ist, übernimmt als Person nicht die Verantwortung für das, was sich im Journalismus tut. " ${ }^{14}$ Andererseits aber setzt er hinzu: "Was in den Journalismus an Persönlichem eingebracht wird, was von der Person folglich auch verletzt werden kann, dafür ist die Person selbst verantwortlich. Die Person ist etwa verantwortlich für Fahrlässigkeit, für subjektive Gefühlsäußerungen, für Eigensinn, für Eigenwilligkeit, etc. Damit verstößt sie gegen die Wert- und Normtreue des Journalismus und sie hat dafür persönlich gerade zu stehen. Persönliches Fehlhandeln wird von journalistischen Berufs- und Arbeitsrollen nicht gedeckt. ${ }^{15}$ Irgendwie scheinen hier Grenzen der systemtheoretischen Analyse zu liegen: Zum einen entleert sie den Journalismus von normativen Elementen und »entpersönlicht« ihn, was zumindest psychologisch fragwürdig ist. Zum anderen wird die Erhaltung und das Funktionieren des Systems selbst zu einer Norm erhoben, die von der »Person « verletzt werden kann.

Trotz solcher erkennbaren Ansätze zu einer Fundierung der journalistischen Berufsethik scheint von diesem Thema bisher aber wenig in die Journalistenausbildung einzumünden. Eigene akademische Lehrveranstaltungen dazu gibt es kaum, was allerdings nicht bedeuten muß, daß hier und da berufsethische Fragen des Journalismus nicht doch aufgegriffen und mit behandelt werden. Doch wird man dem Thema in bloß beiläufiger Form vermutlich kaum gerecht werden können. Sucht man darüber hinaus zu gelangen, so bieten sich für eine Beschäftigung mit journalistischer Berufsethik m.E. drei Ebenen an, eine theoretische, eine empirische und eine pragmatische. Was auf diesen drei Ebenen behandelt werden kann, das soll im folgenden ohne Anspruch auf Vollständigkeit angedeutet werden.

Auf der ersten, theoretischen Ebene kann man wiederum verschiedene Teilaspekte des Themas angehen. Zunächst läßt sich z.B. umreißen, welches die Bedingungen des ethischen Handelns im Journalismus sind. Diese Bedingungen sind vor allem struktureller, juristisch-politischer und ökonomischer Art. Strukturell bedeutsam ist, daß es sich bei der Massenkommunikation bisher im wesentlichen um indirekte und einseitige Kommunikation handelt. Mag es im Ansatz auch eine wechselseitige Rollenübernahme geben, wie sie der symbolische Interaktionismus unterstellt: Die Interaktionspartner bleiben doch getrennt und weitgehend anonym. Dies hat durchaus Konsequenzen für die ethische Dimension des Verhaltens. Arnold Gehlen hat nämlich darauf hingewiesen, daß bestimmte menschliche Reaktionen auf $\gg \mathrm{Nahsicht}$ « eingestellt sind und daß eine "ethische Verunsicherung als Folge der Durchbrechung der Nahsicht-Bedingung ${ }^{16}{ }^{16}$ eintritt. Einfacher gesagt, man ist weniger bereit, ethische Verpflichtungen gegenüber nicht anwesenden Partnern einzuhalten. ${ }^{17}$ Die Massenkommunikation erfordert, so Gehlen, eine »Fern-Ethik «, weil im Vergleich zur personalen Kommunikation die Handlungsfolgen nicht unmittelbar zu beobachten sind und auch die Hemmungsschwellen anders liegen. Dabei gilt dies nicht nur hinsichtlich der Adressaten der journalistischen Berichterstattung, sondern auch bezüglich der Akteure, die ihre Objekte sind.

Außer strukturellen gibt es juristisch-politische Bedingungen der journalistischen $\mathrm{Be}-$ rufsethik. Ein Bedarf an professioneller Ethik ergibt sich aus den rechtlich vergleichsweise geringen Einschränkungen, denen die Medien unterliegen. ${ }^{18}$ Ethische 
Selbstverpflichtung ist als freiwilliger Akt das Korrelat zur Freiheit, was übrigens nochmals Journalismus und Wissenschaft verbindet, deren Freiheiten in Art. 5 des Grundgesetzes in unmittelbarer Nachbarschaft zueinander stehen. Je größer die Autonomie, desto notwendiger erscheint im Prinzip eine ethische Selbstbindung. Allerdings darf man angesichts dieser Gemeinsamkeit nicht die Unterschiede übersehen, die in der Legitimation und in den internen Regelmechanismen zwischen wissenschaftlicher und journalistischer Freiheit bestehen. Zwar sind journalistische Handlungsmöglichkeiten insofern eingeengt als die Pressefreiheit auch ihre Grenzen hat und im Rundfunk z.B. die Vorgaben des gesetzlichen Programmauftrags hinzutreten. Aber solche formellen Bestimmungen sind das eine, ihre tatsächliche Einhaltung etwas anderes. Deshalb wird man sich auch nicht mit dem Hinweis auf gesetzliche Regelungen (z.B. Sorgfaltspflicht) begnügen können. Sie geben nur einen äußersten Rahmen für die berufsethische Selbstverpflichtung ab, die gerade eine übermäßige Verrechtlichung verhindern soll. Während das Recht ein bestimmtes Verhalten erzwingt bzw. verbietet und dazu Sanktionsformen vorsieht, verlangt die Ethik, freiwillig etwas zu tun oder zu unterlassen, auch ohne daß entsprechende Sanktionsmöglichkeiten bestehen.

Zum dritten sind es ökonomische Bedingungen, denen die journalistische Berufsethik unterworfen ist. Es liegt in der Logik eines Systems, das auf der Meinungs-, Presseund Informationsfreiheit sowie auf der Gewerbefreiheit gegründet ist, daß verschiedene Anbieter miteinander konkurrieren und daß die Nachfrage das Angebot (mit)bestimmt. Dabei sind die Unterschiede, die hier (noch) zwischen Presse und Rundfunk bestehen, nicht prinzipieller, sondern struktureller Art. Auch ist im Pressewesen für sich genommen die Konkurrenz graduell unterschiedlich. Gleichwohl steht journalistisches Handeln hierzulande faktisch mehr oder weniger in einem Wettbewerb, sei es um Auflagenzahlen, sei es um Einschaltquoten. Durch diesen Wettbewerb erfährt die journalistische Berufsethik leicht eine Relativierung. Bestimmte, moralisch möglicherweise problematische Handlungsweisen der Massenmedien mögen gerechtfertigt werden, weil, was man nicht selbst tut, von der Konkurrenz getan wird, oder weil das Publikum offenkundig bestimmte Dinge wünscht und dafür bezahlt. Im Streben nach publizistischem und ökonomischem Erfolg können also Gefahren für die Berufsethik des Journalismus liegen. Für die dabei auftretenden Abwägungen wird man schwerlich allgemeingültige Regeln formulieren können. Aber man muß in der Journalistenausbildung das Spannungsverhältnis von Berufsethik und Marktorientierung bewußt machen. ${ }^{19}$

Auf der hier skizzierten theoretischen Ebene kann es sich aber nicht nur darum handeln, das Bedingungsgefüge der journalistischen Berufsethik einsichtig zu machen. Vielmehr können hier überdies Ansätze der Ethik daraufhin überprüft werden, ob und inwieweit sie auf das journalistische Handeln angewandt werden können. Auch dies läßt sich hier wiederum nur beispielhaft andeuten. Zwei unterschiedliche Typen ethischer Systeme sind die Pflichtethik einerseits und die utilitaristische Ethik andererseits. Die Pflichtethik, die auch deontologische Ethik genannt wird, hat Immanuel Kant vertreten, und er hat ihr im kategorischen Imperativ eine knappe, klassische Form verliehen. Ihr zufolge handelt der Mensch sittlich, wenn er sich nach normativ vorgegebenen Verpflichtungen und allgemeinen Maximen richtet, die in sich gut sind.$^{20}$ Die utilitaristische Ethik, die auch teleologische Ethik heißt und die von Jeremy Bentham und John Stuart Mill begründet wurde, wertet ein Handeln dann als sittlich, wenn es nützlich ist für das allgemeine Wohlergehen, d.h. »das größtmögliche Glück der größtmöglichen Zahl * fördert. ${ }^{21}$ Moralität wird hier also von den Folgen 
bestimmt, zumindest von einem Übergewicht der guten gegenüber den schlechten Folgen. Beide Konzeptionen werfen mehrere Schwierigkeiten auf: Im ersten Fall ist $\mathrm{zu}$ fragen, wie man zu den vorgegebenen Verpflichtungen und Normen gelangt und wie diese in ihrer Allgemeinheit mit dem praktischen Leben des Individuums und der Gesellschaft zu vermitteln sind. Im zweiten Fall stellt sich das Problem der empirischen Kenntnis der Folgen von Handlungen und ihrer Bedeutung für das Wohl der Allgemeinheit bzw. die Frage, wonach man das Wohlergehen bemißt oder was »das größtmögliche Glück « denn sei.

Die alte Unterscheidung zwischen Pflichtethik und utilitaristischer Ethik begegnet uns wieder in Max Webers bekannter Entgegensetzung von Gesinnungsethik und Verantwortungsethik bzw. von wertrationalem und zweckrationalem Handeln. ${ }^{22} \mathrm{Ge}-$ sinnungsethiker ist nach den Worten von Robert Spaemann derjenige, "der bestimmte Handlungen kontextunabhängig als moralisch oder unmoralisch qualifiziert, also ohne Rücksicht auf die Folgen bestimmter Handlungen oder Unterlassungen das tut, was er für das sittlich Gebotene hält ${ }^{23}$, Verantwortungsethiker hingegen derjenige, „der bei seinem Handeln die Gesamtheit der Folgen seines Handelns bedenkt und der die Bewertung dieser Folgen zum Maßstab seiner Handlungen macht. ${ }^{24}$ Neuerdings wird für letzteres auch von einer »konsequentialistischen Ethik « gesprochen.

Lassen sich diese Grundtypen ethischer Orientierung nun auf den Journalismus beziehen und welche scheint dabei angemessener, hilfreicher? Die Beantwortung dieser Frage setzt eigentlich eine Funktionsbestimmung des Journalismus im Rahmen unseres politischen Systems voraus. Diese muß ich mir hier ersparen. Aber ob Pflichtethik und Gesinnungsethik mit den sozialen Funktionen des Journalismus vereinbar sind, ist zumindest fraglich, wenn man bedenkt, daß Max Weber diesen Typ strenggenommen an der Figur des Heiligen festgemacht hat. So mag es naheliegender erscheinen, den Journalismus heute auf eine konsequentialistische Ethik zu verpflichten, d.h. nicht die Absichten der Journalisten, sondern die Verantwortung für die Folgen ihres Handelns zur Richtschnur zu machen.

Aber auch dies wirft wiederum eine ganze Reihe von Problemen auf. Inwieweit lassen sich die Folgen des journalistischen Handelns - wohlgemerkt im System der indirekten, einseitigen Massenkommunikation - überhaupt ermitteln oder gar antizipieren? Für welche Folgen muß der Journalist Verantwortung übernehmen, nur für die beabsichtigten und vorhergesehenen (aber in Kauf genommenen) oder auch für die unvorhergesehenen, insbesondere die Nebenfolgen ? ${ }^{25}$ Und wie läßt sich mit solcher Verantwortung ernst machen? Angesichts dieser Fragen könnte vielleicht ein Defätismus aufkommen, und in der Tat ist es ein noch häufig geäußertes Argument, über die Folgen des Journalismus wisse man gar nichts Gesichertes. Dagegen könnte jedoch die Wirkungsforschung durchaus Abhilfe leisten, obwohl in ihr noch vieles ungesichert und umstritten ist. Eine andere Gefahr liegt darin, daß die konsequentialistische Ethik die Verantwortung überzieht, als sei der Mensch oder der Journalist für alles, ja für die Welt als ganze verantwortlich. Gegen solche Universalverantwortung hat Spaemann für eine »gestufte Verantwortung " plädiert, denn: »Die überdehnte Verantwortungsethik führt in Wirklichkeit in die Unverantwortlichkeit reiner Gesinnungsethik. « ${ }^{26}$ Überdies, und das hat schon Max Weber selbst eingeräumt, sind Gesinnungsethik und Verantwortungsethik keine strengen Gegensätze, »sondern Ergänzungen, die zusammen erst den echten Menschen ausmachen ${ }^{2} .{ }^{27}$ 
Gegen die Ethik in ihrer herkömmlichen Form wird immer wieder eingewandt, sie gehe bloß von Vorstellungen aus, wie der Mensch sein soll, ohne zu fragen, was er ist oder was er tut. Diesem Vorwurf läßt sich begegnen, indem man nach der faktischen Verwirklichung des Sollens bzw. der Ethik fragt. ${ }^{28}$ Damit gelangen wir zur zweiten, empirischen Ebene in der Behandlung der journalistischen Berufsethik. Hier interessiert, welchen Normen und Werten Journalisten bei ihrer Arbeit tatsächlich folgen, welche beruflichen Verhaltensweisen und Mittel sie für gerechtfertigt oder ungerechtfertigt halten, inwieweit sie bereit sind, Verantwortung zu übernehmen oder diese ablehnen. Dabei soll das Maß an Kongruenz zwischen bestimmten ethischen Normen und der Realität im Journalismus ermittelt werden. Zwar werden Normen im Prinzip nicht dadurch hinfällig, daß sie nicht befolgt werden. Aber Abweichungen zwischen Normen und Wirklichkeit dürfen nicht beliebig groß werden, will man nicht in eine völlige Unverbindlichkeit geraten. Und solche Brüche aufzudecken, kann den Überlegungen zur journalistischen Berufsethik zumindest den notwendigen Realitätsgehalt verschaffen.

Bei der Durchsicht der Journalismusforschung stößt man durchaus auf eine Reihe von empirischen Studien zu Fragen der journalistischen Berufsethik. Auch lassen sich hier die Untersuchungen zum Selbstbild und zum Rollenverständnis von Journalisten hinzuziehen. Allerdings bedeutet dies nicht, daß man bereits eine hinreichende Datenbasis hätte. Auch empirisch ist die journalistische Berufsethik nur bruchstückhaft erkundet, so daß sich der Forschung noch weitere Aufgaben stellen. Zudem ist die Mehrzahl der Studien amerikanischer Herkunft. So nützlich diese sind, so steht doch dahin, ob und inwieweit ihre Ergebnisse auf die Journalisten in der Bundesrepublik übertragbar sind. Denn bei diesem Thema können zwischen verschiedenen Ländern bemerkenswerte Unterschiede bestehen. Renate Köcher hat in ihrer Untersuchung z.B. ermittelt, daß englische Journalisten in weit größerem Maße als deutsche bereit sind, bestimmte Grenzen bei der Informationsbeschaffung zu übertreten. ${ }^{29}$

Die Ergebnisse der vorliegenden empirischen Studien zur journalistischen Berufsethik hier im ganzen zu resümieren, ist nicht möglich. ${ }^{30}$ Doch soll auf einige Befunde zu verschiedenen Normbereichen journalistischen Handelns beispielhaft hingewiesen werden. Wie sich zeigt, gibt es Berufsnormen, bei denen die Journalisten in ihrem Urteil fast ausnahmslos übereinstimmen, während andere unterschiedlich und kontrovers bewertet werden. Vereinzelt hat man zum Vergleich auch untersucht, wie die Bevölkerung gewisse journalistische Handlungsweisen einschätzt.

Was die Informationsbeschaffung angeht, haben Whitlow und van Tubergen in einer mehr explorativen Studie drei Typen von Journalisten ermittelt. ${ }^{31}$ Während es dem einen Typ nicht darauf ankommt, um jeden Preis zu einem Bericht zu gelangen, sieht der andere darin die wesentliche Aufgabe seines Berufes und ist dementsprechend auch bereit, alle erdenklichen Mittel einzusetzen (z.B. Angabe einer falschen Identität, Drohung mit öffentlicher Bloßstellung, Verwendung gefälschter Dokumente, Benutzung eines Abhörgeräts). Ein dritter Typ steht in seinen Wertungen zwischen den beiden anderen. Nach der bereits erwähnten Studie von Renate Köcher weisen englische Journalisten eine große Ähnlichkeit zum zweiten Typ auf. Über 70 Prozent von ihnen zeigten sich bereit, alle in der Befragung vorgegebenen unlauteren Mittel der Informationsbeschaffung einzusetzen. Hingegen billigten von den deutschen Journalisten 25 Prozent für Informationen Geldbeträge zu bieten (»Scheckheft-Journalismus«), 22 Prozent eine falsche Identität vorzugeben und 8 Prozent Informationen 
unter Druck zu setzen. Offenbar neigen in der Bundesrepublik jüngere Journalisten mehr zu fragwürdigen Mitteln der Informationsbeschaffung als ihre älteren Kollegen. $^{32}$

Nahezu einstimmig gilt den Berufsangehörigen die Wahrhaftigkeit als eine wichtige Eigenschaft, um ein guter Journalist zu sein. ${ }^{33}$ Andererseits bestehen aber Zweifel, ob man diesem Anspruch genügen kann. Insbesondere die Forderung nach Objektivität wird für schwer erfüllbar gehalten. Auch die Sorgfaltspflicht wird als solche weitgehend anerkannt. Doch sind Journalisten großenteils bereit, angesichts von großem Aktualitätsdruck und hohem Nachrichtenwert der berichteten Ereignisse die Sorgfaltspflicht hintanzustellen. ${ }^{34}$ Das Verlangen nach vollständiger Berichterstattung findet ebenfalls weitgehend Zustimmung, aber auch hier wird eingeräumt, daß Unterdrückung von Nachrichten vorkommt. Mehrheitlich lehnen die Journalisten vor allem das Weglassen von Nachrichten aus Rücksicht auf Inserenten ab. ${ }^{35}$

Einen hohen Wert hat für Journalisten der Informantenschutz. Hier ist man sich besonders einig. ${ }^{36}$ Dem Informantenschutz wird von einem erheblichen Teil der Journalisten selbst dann Vorrang eingeräumt, wenn eine Unterrichtung der Polizei Sachoder Personenschäden verhindern könnte. Im Konfliktfall den Informanten zu dekken, dafür sprachen sich 54 Prozent der deutschen Journalisten aus, 25 Prozent erklären sich bereit, gegebenenfalls der Polizei einen Hinweis zukommen zu lassen. ${ }^{37}$

Ein wesentliches Spannungsbild für die journalistische Berufsethik besteht zwischen Öffentlichkeitsprinzip und Persönlichkeitsschutz. Befragungsergebnisse liegen hier primär für die Kriminalitäts- und Gerichtsberichterstattung vor. ${ }^{38}$ Bei jugendlichen und noch nicht verurteilten Angeklagten wird der Persönlichkeitsschutz eher berücksichtigt als bei rechtskräftig Bestraften. Doch ist damit nur ein Teilbereich des Persönlichkeitsschutzes angesprochen. Im Verhältnis dazu wird der Informationsanspruch der Öffentlichkeit dann für wichtiger gehalten, wenn hohe Nachrichtenwerte ins Spiel kommen. Die Benutzung geheimer Regierungsunterlagen hielten übrigens 57 Prozent der deutschen Journalisten für akzeptabel. ${ }^{39}$

Sich als Journalist bestechen zu lassen, gilt herkömmlich als schwerer Verstoß gegen die journalistische Berufsethik. Allerdings scheint zumindest bei amerikanischen Journalisten die Bereitschaft, Vergünstigungen anzunehmen, gewachsen zu sein. Hier haben sich offenbar Umgangsformen eingebürgert, die nicht mehr rundweg abgelehnt werden (können). Für besonders verlockend oder gefährlich hielten die Befragten einer amerikanischen Studie das Angebot einer Auslandsreise. Interessant ist, daß Journalisten sich selbst mehr als ihren Kollegen zutrauen, trotz Vergünstigungen objektiv zu bleiben. ${ }^{40}$

Zu den ehrwürdigen journalistischen Berufsnormen gehört die Trennung von Nachricht und Meinung. Wie schon die Untersuchung von Schönbach gezeigt hat, wird diese Norm aber nur zum Teil konsequent befolgt. ${ }^{41}$ Allgemeingültigkeit besitzt sie jedenfalls nicht. Nicht nur von der gewählten Darstellungsform, sondern vor allem vom Typ der Zeitung und vom journalistischen Selbstverständnis hängt es ab, ob auf die Trennung von Nachricht und Meinung geachtet wird bzw. inwieweit beide synchronisiert werden.

Die Frage der journalistischen Verantwortungsbereitschaft haben schließlich Kepplinger und Vohl in einer Befragung von ZDF-Journalisten untersucht. Dabei zeigte sich ein auffälliger Bruch: »Während durchschnittlich 86 Prozent der befragten Re- 
dakteure des ZDF moralische Verdienste für die positiven Folgen journalistischer Berichte beanspruchten, sind nur durchschnittlich 25 Prozent von ihnen bereit, eine moralische Verantwortung für die negativen Folgen ihrer journalistischen Berichte zu übernehmen. ${ }^{42}$ Die Autoren sprechen hier von einem asymmetrischen Verhältnis: "Dem Anspruch auf moralische Verdienste entspricht keine Bereitschaft zur Übernahme moralischer Verantwortung. ${ }^{43}$ Allerdings fragt sich, in wieweit es sich bei dieser Asymmetrie bezüglich positiver und negativer Folgen um ein spezifisch journalistisches oder ein allgemein menschliches Phänomen handelt.

Die Geltung journalistischer Berufsnormen durch Befragungen empirisch zu ermitteln, ist nicht ganz unproblematisch. Denn ihre verbale Anerkennung und ihre tatsächliche Befolgung können zweierlei sein. So ist das grundsätzliche Bekenntnis zur Verantwortung auch nicht identisch mit der Bereitschaft, im konkreten Fall für eintretende Folgen die Verantwortung wirklich zu übernehmen. Im übrigen muß, ja darf die empirische Analyse nicht dabei stehen bleiben, die berufsethische Orientierung der Journalisten bloß zu registrieren. Die ermittelten Befunde sind vielmehr kritisch an den Normen selbst zu messen. Insofern geht es auf der empirischen Ebene keineswegs um eine Schwächung, sondern durchaus um eine Stärkung der journalistischen Berufsethik.

Indessen wird sich die Journalistenausbildung mit der empirischen Analyse berufsethischer Fragen des Journalismus noch nicht begnügen. Sie wird vielmehr auf eine dritte, pragmatische Ebene übergreifen, zumindest dort, wo die Ausbildung journalistisch-praktische Anteile besitzt. Zwar können journalistische Berufsnormen, wie sie hier z.T. erwähnt wurden und wie sie zahlreicher noch in den Berufskodices oder in Programmrichtlinien enthalten sind, auch einer theoretischen Analyse unterzogen werden. So lassen sich beispielsweise das Objektivitätspostulat oder die Forderung, Nachricht und Meinung zu trennen, aus erkenntnistheoretischer oder wahrnehmungspsychologischer Sicht analysieren..$^{44}$ Aber ihr Problemgehalt dürfte nur am praktischen Beispiel voll erfahrbar werden. Was Sorgfaltspflicht ist, läßt sich schwerlich (nur) theoretisch vermitteln. Man muß es in der Recherche, bei der Beschaffung von Information lernen, wozu schon die wissenschaftliche Informationssicherung taugt. ${ }^{45}$ Doch kann Sorgfalt erst recht auch in der journalistischen Recherche eingeübt werden. Nicht alle Normen lassen sich freilich wie diese in der Simulation erproben.

Auf dieser dritten Ebene geht es gleichsam um die "handwerklichen « Aspekte der journalistischen Berufsethik bzw. um die ethischen Aspekte des journalistischen "Handwerks «. Die handwerklichen Mittel des Journalismus sind Sprache und Bild. Welche ethischen Dimensionen, so ist zu fragen, haben z.B. Sprachgebrauch und Wortwahl, aber auch Bildwahl und Bildausschnitt, Kameraperspektive, Beleuchtung und Schnitt? Dabei wird man von der pragmatischen wieder auf die empirische Ebene zurückgeführt werden, so weit solche ethischen Fragen die Klärung der Wirkungsweisen der journalistischen Darstellungsmittel verlangen. Auch eine Norm wie die Trennung von Nachricht und Meinung, die als regulative Idee auch dann nicht aufgegeben werden sollte, wenn sie faktisch nur begrenzt befolgt wird und erfüllbar ist, kann durch eine Schärfung des Sprachbewußtseins gestärkt werden. Denn nicht immer wird sie bewußt verletzt, sondern, wie mir Erfahrungen in der Ausbildung zeigen, nur deshalb, weil man den Unterschied zwischen einer Faktenwiedergabe und einer Meinungsäußerung bzw. zwischen Referat und Argument gar nicht erkennt. 
Drei Ebenen habe ich hier genannt, auf denen man sich in der Journalistenausbildung mit der journalistischen Berufsethik beschäftigen kann. Alle drei gehören im Grunde zusammen und müssen einander ergänzen. Dabei dürften die theoretische und die empirische Analyse ihren Platz innerhalb wissenschaftlicher Lehrveranstaltungen haben, die pragmatische Behandlung eher in die berufspraktischen Übungen gehören. Auch wenn man hier gar nicht von Ethik spricht, geht es implizit doch oft um moralische Fragen.

Abschließend seien noch einige Einschränkungen gemacht. Natürlich kann es nicht darum gehen, eine homogene Ethik für das gesamte Mediensystem zu etablieren. Aber wenn die Gesellschaft Verhaltensregeln von mehr als nur relativer Gültigkeit braucht, so gilt dies doch auch für den Journalismus. Beide sind überdies nicht unabhängig voneinander. Können die Massenmedien moralisch besser sein als die Gesellschaft, kann man von jenen mehr Moral verlangen als von dieser, so hat man gefragt. Angesichts der Rückbindung an die Nachfrage, von der ich gesprochen habe, mag man hier seine Zweifel haben. Aber der Hinweis auf die "Gesellschaft" kann auch nicht alles entschuldigen oder rechtfertigen. Immerhin zeigen die empirischen Daten z.B. erhebliche Unterschiede in den Auffassungen von Journalisten und Bevölkerung. Und die Gesellschaft selbst stellt eine Resultante auch der Medienwirkung dar. Gleichwohl folgt aus diesem Zusammenhang, daß die journalistische Berufsethik künftig nicht zuletzt davon abhängt, welche Ethik sich die Gesellschaft bewahrt und von Generation zu Generation weitergibt. Endlich sei noch einem Mißverständnis vorgebeugt: Ziel der Einbeziehung journalistischer Berufsethik in die Journalistenausbildung kann und soll nicht sein, den künftigen Journalisten einen starren Normenkatalog mit auf den Weg zu geben. Es kann allenfalls darauf ankommen, ihr Bewußtsein von den ethisch-moralischen Dimensionen journalistischen Handelns zu wecken und Kriterien zu vermitteln, die für die immer wieder zu treffenden Entscheidungen bedeutsam sein können.

\section{Anmerkungen}

1 Vortrag, gehalten auf der 32. Jahrestagung der Deutschen Gesellschaft für Publizistik- und Kommunikationswissenschaft vom 8. - 10. Mai 1987 in Eichstätt zum Thema "Zwischenbilanz der Journalistenausbildung ". Abdruck auch in dem die Tagung unter gleichem Titel dokumentierten Sammelband, Verlag Ölschläger, München

2 Vgl. Emil Dovifat: Die Gesinnungen in der Publizistik. In: Erich Feldmann (Hrsg.): Film und Fernsehen im Spiegel der Wissenschaft. Gütersloh 1963. S. 25-41. - Dazu vgl, auch Elisabeth Noelle-Neumann: Gesinnung und Verantwortung. In: Kurt Koszyk, Volker Schulze (Hrsg.): Die Zeitung als Persönlichkeit. Festschrift für Karl Bringmann. Düsseldorf 1982. S. 23-28.

3 Vgl. z.B. Elisabeth Noelle-Neumann in einem Diskussionsbeitrag in: Eugen Biser u.a.: Die Medien - das letzte Tabu der offenen Gesellschaft. Die Wirkung der Medien auf Politik und Kultur. Mainz 1986. S. 146.

4 Vgl. Hermann Boventer: Ethik und Journalismus: Eine Untersuchung des Hastings Center zur Medienethik im Ausbildungsprogramm an amerikanischen Colleges und Universitäten. In: Communicatio Socialis 15 (1982) S. 329-333.

5 Vgl. N.A. Crawford: The Ethics of Journalism. New York 1924. - W.F. Gibbons: Newspaper Ethics: A Discussion of Good Practice for Journalists. Ann Arbor 1926. - A.F. Henning: Ethics and Practices in Journalism. New York 1932. - Lee Thayer (Hrsg.): Communication: Ethical and Moral Issues. London, New York, Paris 1973. - Ders.: Ethics, Morality and the Media. Reflections on American Culture. New York 1980. - John C. Merrill: The Imperative of Freedom. A Philosophy of Journalistic Autonomy. New York 1974. - John C. 
Merrill, Ralph D. Barney: Ethics and the Press. Readings in Mass Media Morality. New York 1975. - John L. Hulteng: The Messenger's Motives ... Ethical Problems of the News Media. Englewood Cliffs 1976. - Clifford G. Christians: Beyond Quandries: A Plea for Normative Ethics. In: Mass Communication Review 6 (1979) S. 28-31. - Bruce M. Swain: Reporters' Etics. Ames 1978. - William L. Rivers, Wilbur Schramm, Clifford G. Christians: Responsibility in Mass Communication. 3rd Edition. New York u.a. 1980. - John M. Phelan: Disentchantment. Meaning and Morality in the Media. New York 1980. - Clifford G. Christians, Kim B. Rotzoll, Mark Fackler: Media Ethics. Cases and Moral Reasoning. New York, London 1983. - H. Eugene Goodwin: Groping for Ethics in Journalism. Ames 1983. - Tom Goldstein: The News at any Cost. How Journalists compromise their Ethics to shape the News. New York 1985. - Deni Elliott (Hrsg.): Responsible Journalism. Beverly Hills u.a. 1986. Als Überblicke vgl.: Clifford G. Christians: Fifty Years of Scholarship in Media Ethics. In: Journal of Communication 27 (1977) H. 4 S. 19-29. - Hermann Boventer: Journalistenmoral als »Media Ethics«. Kodifizierte Pressemoral und Medientechnik in den Vereinigten Staaten von Amerika. In: Publizistik 28 (1983) S. 19-39.

6 Vgl. besonders Hans Jonas: Das Prinzip Verantwortung. Versuch einer Ethik für die technologische Zivilisation. Frankfurt/M. 1979. - Felix Hammer: Selbstzensur für Forscher? Schwerpunkte einer Wissenschaftsethik. Zürich 1983. - Elisabeth Ströker (Hrsg.): Ethik der Wissenschaften? Philosophische Fragen. München, Paderborn 1984. - Hans Michael Baumgartner, Hansjürgen Staudinger (Hrsg.): Entmoralisierung der Wissenschaften? München, Paderborn 1985.

7 Alfons Auer: Verantwortete Vermittlung. Bausteine einer medialen Ethik. In: Ethik und Kommunikation. Hohenheimer Medientags 1980. Hrsg. von der Deutschen Bischofskonferenz und der Katholischen Akademie. Stuttgart 1980. S. 64-86. Zitat hier S. 65. - Vgl. ferner ders.: Verantwortete Vermittlung. Bausteine einer Informationsethik des Rundfunks. In: Stimmen der Zeit 104 (1979) S. 15-24. - Ders.: Ist Unterhaltung vertane Zeit? Überlegungen zur Unterhaltung in den Massenmedien aus der Sicht einer theologischen Ethik. In: Stimmen der Zeit 105 (1980) S. 735-749.

8 Vgl. Hermann Boventer: Ethik des Journalismus. Ansätze und Fragestellungen. In: Stimmen der Zeit 108 (1983) S. 387-401. - Ders.: Ethik des Journalismus. Zur Philosophie der Medienkultur. Konstanz 1984. - Ders.: Das Prinzip Verantwortung in der Massenkommunikation. Problem einer kommunikationswissenschaftlich fundierten Ethik des Journalismus. In: Hans Maier (Hrsg.): Ethik der Kommunikation. Freiburg (Schweiz) 1985. S. 53-72. - Zur Kritik daran vgl. u.a. die Rezension von Wolfgang Wunden in: Communicatio Socialis 18 (1985) S. $195 f$.

9 Ethik des Journalismus (1984) S. 18.

10 Ethik des Journalismus (1983) S. 397

$11 \mathrm{Vgl.} \mathrm{Manfred} \mathrm{Rühl:} \mathrm{Ethik} \mathrm{und} \mathrm{Humankommunikation.} \mathrm{Überlegungen} \mathrm{zu} \mathrm{ihrer} \mathrm{kommunika-}$ tionswissenschaftlichen Begründung. In: Georg Wodraschke (Hrsg.): Jugendschutz und Massenmedien. München 1983. S. 71-86. - Ders.: Ethik - ein Gegenstand der Kommunikationsforschung? In: Ethik und Kommunikation. Hohenheimer Medientage 1980. Hrsg. von der Deutschen Bischofskonferenz und der Katholischen Akademie. Stuttgart 1980. S. 29-49. - Ulrich Saxer: Publizistische Ethik und gesellschaftliche Realität. In: Communicatio Socialis 3 (1970) H. 1 S. 24-39. - Ders.: Journalismus- und Medienethik: Möglichkeiten und Grenzen ethischer Selbstverpflichtung. In: Media Perspektiven $\mathrm{H}$. 1/1984 S. 21-32. - Ders.: Journalistische Ethik eine Chimäre? In: Hans Maier (Hrsg.): Ethik der Kommunikation. Freiburg (Schweiz) 1985. S. 43-52. - Ders.: Konstituenten einer Medien- und Journalismus-Ethik. In: Zeitschrift für evangelische Ethik 30 (1986) S. 21-45. Manfred Rühl, Ulrich Saxer: 25 Jahre Deutscher Presserat. Ein Anlaß für Überlegungen zu einer kommunikationswissenschaftlich fundierten Ethik des Journalismus und der Massenkommunikation. In: Publizistik 26 (1981) S. 471-507.

12 Vgl. Manfred Rühl, Ulrich Saxer a.a.O. S. 487.

13 Hermann Boventer: Ethik des Journalismus (1983) S. 395.

14 Manfred Rühl: Ethik - ein Gegenstand der Kommunikationsforschung? a.a.O. S. 44.

15 Ebd. S. 45. 
16 Arnold Gehlen: Moral und Hypermoral. Eine pluralistische Ethik. Frankfurt/M., Bonn 1969. S. 56.

17 In diesem Zusammenhang hat Gehlen übrigens vermutet, das "Fernsehen, das uns die Leiden der unglücklichen Bevölkerung von Sonstwoland unmittelbar ins Haus setzt, (schaffe) eine noch schwer übersehbare, sicher folgenreiche Veränderung unserer Verpflichtungsgefühle, vielleicht in Hinsicht der A bstumpfung nun auch gegen leibhaft gegenwärtige Leiden.«(ebd.)

$18 \mathrm{Vgl}$. Ulrich Saxer: Journalismus und Medienethik ... a.a.O.

19 Vgl. dazu z.B. Manfred Rühl: Markt und Journalismus. In: Manfred Rühl, Jürgen Walchshöfer (Hrsg.): Politik und Kommunikation (= Festgabe für Franz Ronneberger zum 65. Geburtstag). Nürnberg 1978. S. 237-271.

20 Vgl. Immanuel Kant: Metaphysik der Sitten. Hrsg. von Karl Vorländer. Hamburg 1966. S. $217 \mathrm{ff}$.

21 Vgl. Otfried Höffe: Einführung in die utilitaristische Ethik. München 1979. - Ders. : Sittlich-politische Diskurse. Frankfurt/M. 1981.

22 Vgl. Max Weber: Politik als Beruf. In: M.W.: Gesammelte politische Schriften. 2. erw.Aufl. hrsg. von Johannes Winckelmann. Tübingen 1958. S. 493-548.

23 Robert Spaemann: Wer hat wofür Verantwortung? Der Streit um deontologische und teleologische Ethik. In: Herder Korrespondenz 36 (1982) S. 345-350, 403-308. Zitat hier S. 345.

24 Ebd. Vgl. dazu auch Günther Patzig: Noch einmal: „Gesinnungsethik « und „Verantwortungsethik «. In: Bernd Rebe, Klaus Lompe, Rudolf von Thadden (Hrsg.): Idee und Pragmatik in der politischen Entscheidung. Alfred Kubel zum 75. Geburtstag. Bonn 1984. S. 37-42.

25 Vgl. dazu Robert Spaemann: Nebenwirkungen als moralisches Problem. In: R.S.: Zur Kritik der politischen Utopie. Stuttgart 1977.

26 Robert Spaemann a.a.O. S. 408.

27 Max Weber: Politik als Beruf a.a.O. S. 547.

$28 \mathrm{Vgl}$. zu dieser Grundsatzfrage Bernhard Schleißheimer: Sein und Sollen. Zur Frage der theoretischen Begründbarkeit praktischer Normen. München 1978.

29 Vgl. Renate Köcher: Spürhund und Missionar. Eine vergleichende Untersuchung über Berufsethik und Aufgabenverständnis britischer und deutscher Journalisten. Diss. München 1985.

30 Eine erste, vorläufige Übersicht bietet Ilona Bernardy: Empirische Studien zur journalistischen Berufsethik. Magisterarbeit. Mainz 1984.

31 Vgl. S. Scott Whitlow, G. Norman van Tubergen: Patterns of Ethical Decisions among Investigative Reporters. In: Mass Communication Review 6(1978/79) S. 2-9.

$32 \mathrm{Vgl}$. Renate Köcher a.a.O. S. $141 \mathrm{ff}$

33 Dem stimmten 96 Prozent der britischen und 94 Prozent der deutschen Journalisten zu. Vgl. Renate Köcher a.a.O. S. $131 \mathrm{ff}$.

34 Vgl. Renate Köcher a.a.O. S. 140 ff. - Reinhart Stalmann: Über die Professionalisierungstendenzen bei den Pressejournalisten in der BRD. Diss. Zürich 1974. - J. Herbert Altschull: The Amoral Morality of Editors. Uniformity and the Nose for the Camel. In: Anne van der Meiden (Hrsg.): Ethics and Mass Communication. Utrecht 1980. S. 89-107. - Donald S. Weinthal, Garrett J. O'Keefe jr.: Professionalism among Braodcast Newsmen in an Urban Area. In: Journal of Broadcasting (1974) S. 193-209.

$35 \mathrm{Vgl}$. Reinhart Stalmann a.a.O. S. 94.

36 Vgl. Renate Köcher a.a.O. S. 145 ff. - Vince Blasi: The Newsman's Privilege: An Empirical Study. In: Michigan Law Review 70 (1971/72) S. 229-284. - Jack M. McLeod, Searle E. Hawley: Professionalization among Newsmen. In: Journalism Quarterly 41 (1964) S. 529539. - Donald S. Weinthal, Garrett J. O'Keefe a.a.O. - S. Scott Whitlow, G. Norman van Tubergen a.a.O. - David Gordon: Chicago Journalist's and Ethical Principles. In: Mass Communication Review 6 (1979) S. 17-20.

37 Renate Köcher a.a.O. S. 147.

$38 \mathrm{Vgl}$. Hans Mathias Kepplinger, Inge Vohl: Mit beschränkter Haftung. Zum Verantwortungsbewußtsein von Fernsehredakteuren. In: Hans Mathias Kepplinger (Hrsg.): Angepaßte Außenseiter. Was Journalisten denken und wie sie arbeiten. Freiburg, München 1979. S. 223259. Hier insbesondere S. 237 ff. - Renate Köcher a.a.O. S. 150 ff. - Helmut Kerscher: Gerichtsberichtserstattung und Persönlichkeitsschutz. Eine empirisch-rechtspolitische Studie über Entstehung und Wirkung identifizierender Gewaltdarstellung. Diss. Hamburg 1982. 
$39 \mathrm{Vgl}$. Renate Köcher a.a.O. S. 160.

$40 \mathrm{Vgl}$. Keith P. Snaders, Won H. Chang: Codes - The Ethical Free-for-All: A Survey of Journalist's Opinions about Freebies. Columbia 1977. - Vgl. ferner Jack M. McLeod, Searle E. Hawley a.a.O. - Donald S. Weinthal, Garrett J. O'Keefe a.a.O.

41 Vgl. Klaus Schönbach: Trennung von Nachricht und Meinung. Empirische Untersuchungen eines journalistischen Qualitätskriteriums. Freiburg, München 1977.

42 Hans Mathias Kepplinger, Inge Vohl a.a.O. S. 243.

43 Ebd. S. 244.

44 Vgl. u.a. Jürgen Westerstahl: Objectivity is measurable. In: EBU Review 121 B (1970) S. 1317. - Gaye Tuchman: Objectivity as a Strategic Ritual: Newsmen's Notions on Objectivity. In: American Journal of Sociology 77 (1972) S. 660-670. - Ulrich Saxer: Die Objektivität publizistischer Information. In: Wolfgang R. Langenbucher (Hrsg.): Zur Theorie der politischen Kommunikation. München 1974. S. 206-235. - Lutz Huth: Ereignis, Objektivität und Präsentation in Fernsehnachrichten. In: Politische Medienkunde Bd. 3/1977 S. 103-123. - Günter Bentele, Robert Ruoff (Hrsg.): Wie objektiv sind die Medien? Frankfurt 1982.

45 Vgl. dazu Ulrich Saxer: Recherche als journalistischer Auftrag und Prüfstein. In: Fernsehen und Bildung 10(1976) S. 224-250.

\section{Summary}

Unlike in the United States in germanlanguage countries Media Ethics are not treated very extensively but there seems to be a new awakening. Media Ethics can be treated on three levels: The theoretical, the empirical and the pragmatic level. On the theoretical level one can consider the structural conditions for ethical action, the juridical-political as well as the economic conditions. The empirical level concerns the norms and values underlying journalistic work and responsibility with consequences for news gathering and presentation, in following the truth, protecting the information sources, clear division between news and comment etc. The pragmatic level executes insights from different levels into concrete examples of journalistic work. All three levels of Media Ethics are interrelated and complementary.

\section{Résumé}

Contrairement aux Etats-unis d'Amerique l'éthique des médias dans les pays à langue allemande n'est pas traitée d'une facon détaillée. L'éthique des médias peut être traitée à trois niveaux différents: le niveau théorique, empirique et pragmatique. Au niveau théorique on peut considérer les conditions structurelles pour un agissement éthique. Aussi les conditions juridico-politiques que économiques. Le niveau empirique se rapporte aux normes et aux valeurs, qui se fondent sur le travail journalistique et la responsabilité et qui ont des conséquences pour la collection d'information et la présentation, de facon que la fidélité de la verité soit poursuivie, les sources d'information soient protégées et une précise séparation entre l'information et le commentaire se passe. Au niveau pragmatique, les apercus de différents niveaux sont transformés en exemples concrets de travail journalistique. Tous les trois niveaux de l'éthique de médias se trouvent en relation de changement et se complètent.

\section{Resumen}

En contraste a los Estados Unidos no se trata a fondo la ética de los medios en paises de lengua alemana, pero según parece hay un despertar nuevo. Se puede tratar la ética de los medios en tres niveles diferentes: El nivel teórico, empirico y pragmático. En el nivel teórico se puede considerar las condiciones estructurales para el acto ético, no sólo las condiciones juridico-politicas sino también las condiciones de economia. El nivel empirico se refiere a las normas y a los valores que forman la base del trabajo y de la responsabilidad periodistica y que tienen consecuencias respecto a recoger material informativo y a presentarlo al quedar conforme a la verdad, al proteger las fuentes de información, al separar claramente noticias y comentarios etc. En el nivel paragmático el conocimiento de tres niveles diferentes llega a ser un ejemplo concreto del trabajo periodistico. Los tres niveles de la ética de los medios están en relaciones mutuas y se completan. 